be expected, the local effects due to the screening wires have disappeared; but since the discrete patch of cathode illumination still moves over the cathode, there is a steady change of response. The effect of using an aperture which is sufficiently small to give rise to a nearly spherical wave is shown by curve $B$, which was obtained with an aperture (supplied by the Smethurst High Light Co.) of apparent diameter $2 \mu$. In this case not only have the local shading effectis been eliminated, but also the overall systematic variation of response does not occur. To obtain this result it was necessary to bring the source nearer the aperture, greater brilliance being impracticable and increased dynode voltage being undesirable; but the relationship between the significant dimensions was not altered greatly (the cathode patch would have been $0.35 \mathrm{~mm} . \times 0.09 \mathrm{~mm}$. by geometrical theory, which would be quite small enough to lead to detectable effects). It is therefore clear that the use of a sufficiently small aperture can provide a suitable basis for accurate photometry. In curve $B$ some variation of response remains, but after correcting for inverse square law variation $(0.4$ per cent) and cosine law variation $(0.2$ per cent) the response of the photometer is constant to within \pm 1 per cent. On repetition of the experiment, but with the source displaced so as to tend to move the image across the cathode, the same result was obtained.

The results are of importance in deriving spatial frequency responses from direct measurements of the intensity distributions in 'point' images formed by lenses under test. If the photometer aperture is large, a real image of the last surface of the lens under test will be formed by pin-hole camera action on the cathode and, on scanning the point image, the cathode patch will move (and otherwise change in the presence of zonal aberrations), as P. D. Carman has pointed out to me. Taking the inclusion of tho first Fresnel zone as the criterion for the pin-hole camera effect, the aperture should then have a diameter of approximately $160 \mu$, for $\lambda=0.5 \mu$ and $A C=12.7 \mathrm{~mm}$. This is much larger than the value of $5 \mu$ at which some vestige of geometrical image formation has been found to remain; though clearly the image on the cathode must then have been quite diffuse. When the aperture is reduced to a size which includes only $(1 / 80)^{2}$ of the first Fresnel zone, or the diameter is 4 wave-lengths in the photometer described here, no noticeable pin-hole camera effect remains and a reliable, simple photometer is obtained.

Royal Aircraft Establishment,

$$
\text { J. M. NAISH }
$$

Farnborough, Hants.

$$
\text { Nov. } 9 .
$$

${ }^{1}$ Saunderson, Caldecourt and Peterson, J. Opt. Soc. Amer., 35, 681 (1945).

- Naish, J. M., J. Sci. Instr., 28, 138 (1951).

${ }^{3}$ Baker, L. R., J. Sci. Instr., 32, 418 (1955).

- Kessler and Wolfe, J. Opt. Soc. Amer., 37, 133 (1947).

\section{The General Theory of Relativity and Newton's Law of Gravitation}

IT is generally believed that the general theory of relativity is a theory of gravitation which treats only the case for which the Newtonian attraction between particles $m_{1}, m_{2}$ at a distanee $d$ apart is $\gamma m_{1} m_{2} / d^{2}$, where $\gamma$ is a constant, and that the case when $\gamma$ is replaced by a variable quantity $\gamma^{\prime}$ is beyond the scope of the theory. I do not believe that this is the case, and in the following argument I endeavour to show that the general theory of relativity can, in fact, treat the case when $\gamma^{\prime}$ is a slowly varying function of the time, of the type found in Dirac's cosmology ${ }^{1}$.

Newton's law of gravitation can be derived in two cases from the theory of relativity: (1) for weak static fields of matter at rest; (2) for the field of a homogeneous pressure-free distribution of expanding matter. These cases are usually treated rather differently : in (1) a system of 'local' co-ordinates is chosen so as to make space-time quasi-Minkowskian, and the analogue of Poisson's equation is found from the relativistic equations; and in (2) the equations of motion of matter are derived for a system of 'cosmic' co-ordinates which are then found to have their exact analogue in Newtonian theory ${ }^{2}$. Either case can be used to determine the value of the Einstein constant $x=8 \pi \gamma / c^{2}$. Usually (1) is used for this purpose, but it would seem to be preferable to define $x$ by $(2)$ and to derive the local field by joining a centrally symmetric field on to the field of the expanding universe. I have found that when this is done, the 'local' distance is the same as 'cosmie' distance, and 'Iocal' time is approximately the same as 'cosmic' time. One can therefore say that the general theory of relativity exhibits Newton's law of gravitation with constant $\gamma$ in 'cosmic' co-ordinates.

If one now replaces 'cosmic' distance $\rho$ by a new distance $r=\lambda \rho$ ('atomic' distance), where $\lambda$ is a slowly varying function of the time, one finds that the relativistic equations have the Newtonian form for the previous cases (1) and (2), when $\gamma$ is replaced by $\gamma^{\prime}$, a slowly varying function of the time. If one then introduces the condition that $\gamma^{\prime}$ shall have the same value for (1) and (2), in order to ensure that the law of gravitation is universal, it is found that $\gamma^{\prime}$ varies inversely as the epoch, in agreement with the result found in Dirac's cosmology.

Thus the general theory of relativity may be said to exhibit Newton's law of gravitation in two different forms. In one form 'cosmic' distance corresponds to Newtonian distance and $\gamma$ is a universal constant; in the other form, 'atomic' distance corresponds to Newtonian distance and $\gamma$ is replaced by $\gamma^{\prime}$, which varies inversely as the epoch.

Department of Mathematics, King's College,

Newcastle upon Tyne.

$$
\text { Oct. } 19 .
$$

${ }^{1}$ Dirac, P. A. M., Proc. Roy. Soc., A, 165, 199 (1938).

3 McCrea, W. H., and Milne, E. A., Quart. J. Math. (Oxford), 5, 73 (1934).

\section{Carbonatites in Kivu}

IN $1952^{1}$ one of us described specimens of syenitic and carbonate rocks collected in northern Kivu, near the south-western termination of the Lake Edward Rift, by Borgniez, in $1938^{2}$. The junior author has recently had the opportunity of revisiting the locality and has established the carbonatitic nature of the carbonate rocks.

This carbonatite plug extends a little more than a mile in the Lueshe Valley and is intrusive in pelitic schists of the Urundi-Ruzizi series, striking regionally N. $50^{\circ} \mathrm{W}$. (magnetic). These schists of garnet or cyanite grade show a rather mild type of metamorphism ${ }^{1,3}$ which has scarcely destroyed their sedimentary characteristics. They include, immediately west of the carbonatite, a metamorphosed 